\title{
3 Research Square \\ Bone Health in Children With Risk Factors for Low Bone Mass
}

Berta Magallares ( $\nabla$ bmagallares@santpau.cat)

Hospital de la Santa Creu i Sant Pau

Dacia Cerda

Hospital Sant Joan Despí Moisés Broggi: Hospital de Sant Joan Despi Moises Broggi

Jocelyn Betancourt

Hospital de la Santa Creu i Sant Pau

Gloria Fraga

Hospital de la Santa Creu i Sant Pau

Estefanía Quesada-Masachs

Vall d'Hebron Hospital Universitari: Hospital Universitari Vall d'Hebron

Mireia Lopez-Corbetó

Vall d'Hebron Hospital: Hospital Universitari Vall d'Hebron

Montserrat Torrent

Hospital de la Santa Creu i Sant Pau

Ana Marin

Hospital de la Santa Creu i Sant Pau

Silvia Herrera

Hospital de la Santa Creu i Sant Pau

Ignasi Gich

Hospital de la Santa Creu i Sant Pau

Susana Boronat

Hospital de la Santa Creu i Sant Pau

Samuel Zilberman

University of California System

Jordi Casademont

Hospital de la Santa Creu i Sant Pau

Hector Corominas

Hospital de la Santa Creu i Sant Pau

Jorge Malouf

Hospital de la Santa Creu i Sant Pau 
Keywords: Low Bone Mass for Chronological Age, Childhood Osteoporosis, Bone Fragility, Bone Mineral Density

Posted Date: January 5th, 2022

DOI: https://doi.org/10.21203/rs.3.rs-1170693/v1

License: (1) This work is licensed under a Creative Commons Attribution 4.0 International License. Read Full License 


\section{Abstract}

Purpose: To describe clinical and biological characteristics of pediatric patients with at least one risk factor (RF) for low bone mass for chronological age (LBMca)/childhood osteoporosis (COP) and to assess its influence on bone mineral density (BMD).

Methods: Patients between 2 and 20 years of age with at least 1 RF were recruited. Daily calcium intake, number of previous fractures and other RFs and their distribution among different groups were assessed. Spine and whole body DXA and vertebral morphometry were performed.

Results: 103 patients were included. Mean age was 9.8 years old. $52.4 \%$ were female. Of the RFs, $84.5 \%$ presented insufficient calcium intake, $38.8 \%$ were receiving or had received corticosteroids, $31.1 \%$ were receiving other treatments with osteotoxic potential, $13.6 \%$ led a sedentary lifestyle, $12.6 \%$ presented history of fractures, and up to $8.1 \%$ had hypovitaminosis D. $38 \%$ of the cohort had $2 \mathrm{RFs}, 31 \%$ had $3 \mathrm{RFs}$, $15 \%$ had 4 RFs, and $12 \%$ associated 5 or more RFs. $10.5 \%$ met LBMca criteria and $4.8 \%$ met cOP criteria. $73 \%$ of vertebral BMD was justified by age and hypovitaminosis D (positive effect), and male sex and Hispanic ethnicity (negative effect). $82 \%$ of total body less head BMD was justified by age (positive effect), and Hispanic ethnicity and sedentary lifestyle (negative effect).

Conclusions: Pediatric populations with risk of LBM/cOP have 2 or more risk factors. Up to $10.5 \%$ of children with RFs present LBM and $4.8 \%$ have an unknown cOP. RFs related to changes in BMD are age, sex, sedentary lifestyle, ethnicity, and hypovitaminosis D.

\section{Summary:}

This study deals with low bone mass for chronological age (LBMca) and childhood osteoporosis (cOP), both underecognized conditions. We aim to describe children with risk to suffer those diseases. We found that up to $10.5 \%$ of children with risk factors present LBM and $4.8 \%$ present with cOP.

\section{Introduction:}

Currently, osteoporosis is a prevalent problem whose main manifestations are fractures due to bone fragility [1]. Although these fractures occur mainly in a population older than 50 , in many cases, the causes are present during bone development at early ages [2]. Reaching an adequate peak bone mass during adolescence is essential in order to prevent future complications and to keep an optimal state of bone health to be able to cope with the effects of hormonal deficiencies, senescence, and other factors associated to ageing [3].

At present, the impact each modifiable risk factor (RF) has throughout bone development and their prevalence are unknown. Additionally, the short-term and long-term consequences of RFs that appear during childhood are unknown. Early detection of these RFs along with the study and diagnosis of low bone mass for the chronological age (LBMca) may allow better control at early ages. 
The objective of the study is to describe clinical features and risk factors of a pediatric population with at least 1 risk factor associated to bone fragility, to analyze LBM and childhood osteoporosis (COP) prevalence, and to assess which specific RFs associate to LBM in pediatric patients.

\section{Material And Methods:}

We conducted a multicenter ambispective cohort study. Our study included patients under the age of 21 that were referred to the Pediatric Rheumatology Outpatient Clinic for bone health assessment and presenting with at least one RF for bone fragility. These RFs included: malabsorption syndrome, chronic inflammatory diseases, hematological diseases, endocrinopathies, treatment with drugs with osteotoxic potential (such us: corticosteroids or immunosuppressant drugs), or insufficient calcium intake. Patients who had previously received any bone active treatment were excluded. The study obtained approval by the institutional ethics committee at our center (IIBSP-FRA-2016-11). Patients and/or their legal guardians signed informed consent prior to recruitment.

The following demographic and clinical variables were collected: weight, height, pre-existing conditions and dates of onset, number and location of previous fractures, and concomitant treatments. Average calcium intake (milligrams/day) was calculated by the INDICAD 2001 study test [4]. Physical activity was measured in patients older than 12 by the PAQ-A (Physical Activity Questionnaire for Adolescents), and in patients younger than 12 by the PAQ-C (Physical Activity Questionnaire for Children). For both questionnaires, authenticated versions for Spanish population were used $[5,6]$, and their scales range from 1 (very low level of physical activity) to 5 (high level of physical activity). Data on physical activity were not collected from children under 3 years old because it is not validated before that age.

The laboratory parameters collected were calcemia, phosphoremia, $\mathrm{OH}-25$-vitamin $\mathrm{D}$ concentration (determined by liquid chromatography coupled to tandem mass spectrometry), and calciuria from 6-hour urine collection. Outlier results from the 6-hour urine test were double-checked by a 24-hour calciuria test.

The following data were obtained by dual-energy x-ray absorptiometry (DXA): total body and subtotal body less head BMD and BMD at vertebrae L1-L4, as well as total body and vertebrae L1-L4 Z-score. Height adjustment for vertebral and total body Z-score values was performed for all cases by means of the formulas published by Zemel et al. [7]. Densitometric determinations were obtained using an Hologic Discovery densitometer scanner (Hologic, Inc., Bedford, MA, USA).

Presence or absence of vertebral fractures was analyzed with vertebral morphometry by applying Genant's semi-quantitative scale $[8,9]$.

Statistical analyses were performed with the IBM-SPSS (V26.0) software package. Quantitative variables were presented as mean (standard deviation). Categorical variables were presented as absolute frequencies and percentages. Relationship between categorical variables was assessed with contingency tables and Chi square test, or Fisher's exact test. To evaluate quantitative variables in comparison to a two-grouped categorical one, T-test was used and analysis of variance in the case of more than two 
groups. Mann-Whitney U test was used for non-normally distributed ordinal or quantitative variables in the case of two groups, and Kruskal-Wallis test for more than two groups. Pearson's linear correlation coefficient was used to correlate two quantitative variables. Spearman's correlation coefficient was calculated when one of these variables or both were ordinal or showed clear non-normality. In addition, multiple linear regression models were used. Bivariate models and age-adjusted bivariate models were used for studied variables. $95 \%$ confidence intervals were calculated in clinically relevant results. For all cases, type I error level was $5 \%(a=0.05)$ and a bilateral approximation was used.

\section{Results:}

103 consecutive patients with at least 1 risk factor associated to LBMca development were recruited.

Females represented $52.4 \%$ of the cohort. Average age was 9.8 years, Standard deviation (SD) 4.7 , with a range of 2 to 20 years. Table 1 shows patients' characteristics. 
Table 1

Cohort Characteristics

\begin{tabular}{|ll|}
\hline Age Group (Range in Years) & $\mathbf{n}(\%)$ \\
\hline Pre-school age (2-3) & $9(8.7)$ \\
\hline School age (4-9) & $33(32)$ \\
\hline Adolescence (10-17) & $55(53.4)$ \\
\hline Young adult (18-20) & $6(5.8)$ \\
\hline Diagnoses & \\
\hline Malabsorption/ food allergies & $47(46.6)$ \\
\hline Juvenile Idiopathic Arthritis (JIA) & $18(17.5)$ \\
\hline Nephropathies & $18(17.5)$ \\
\hline Hematological diseases & $7(6.8)$ \\
\hline Vasculitis & $4(3.9)$ \\
\hline Systemic autoimmune diseases & $4(3.9)$ \\
\hline Autoinflammatory diseases & $3(2.9)$ \\
\hline Endocrinopathies & $1(1)$ \\
\hline Ethnicities & \\
\hline Caucasian & $92(79.6)$ \\
\hline Hispanic & $11(10.7)$ \\
\hline Arab-Berber & $7(6.8)$ \\
\hline Others & $3(2.9)$ \\
\hline Anthropometric Characteristics & \\
\hline Height $\leq 3$ rd percentile & \\
\hline Height $\geq 97$ th percentile & \\
\hline Weight $\leq 3$ rd percentile & \\
\hline Weight $\geq 97$ th percentile & \\
\hline Number of Fractures (n) by patient & \\
\hline 1 & \\
\hline 2
\end{tabular}




\begin{tabular}{|lll|}
\hline Age Group (Range in Years) & $\mathbf{n}(\%)$ \\
\hline 3 or more & $2(1.9)$ & 6 \\
\hline None & $85(82.5)$ & - \\
\hline
\end{tabular}

Out of the group of patients having malabsorption/food allergies, 19 patients presented cow's milk protein allergy (CMPA), 18 patients presented celiac disease, 7 patients had multiple food allergies, and 1 patient had eosinophilic esophagitis. Additionally, 1 patient with Crohn's bowel inflammatory disease and 1 patient with short bowel syndrome were included. Distribution of Juvenile Idiopathic Arthritis (JIA) subtypes were as follows: 9 oligoarticular, 3 polyarticular, 3 enthesitis-related arthritis, 2 psoriatic, and 1 systemic. The observed nephropathies included 14 patients with nephrotic syndrome, 1 with renal tubular acidosis, and 3 with chronic renal failure (one of these patients had previously received a kidney transplant). Among hematological diseases, 2 patients presented lymphomas and 5 patients had acute lymphoblastic leukemia (ALL), 4 out of these had graft-versus-host disease. The systemic autoimmune diseases (SADs) group included 2 patients with systemic lupus erythematosus (SLE), 1 with autoimmune hepatitis, and 1 with eosinophilic fasciitis. Autoinflammatory diseases were ADA2 deficiency, familial Mediterranean fever, and PFAPA syndrome. In the endocrinological disorder category, 1 patient affected by pituitary hypoplasia was included. In 6 cases, patients had more than one diagnoses: 1 patient had type 1 diabetes mellitus (T1D) and celiac disease, 1 patient had T1D and nephrotic syndrome, 1 patient with CMPA had nephrotic syndrome, 1 JIA patient also had multiple food allergies, 1 patient with Crohn's disease had a partial colectomy, and 1 patient with ALL also presented hypopituitarism.

At some timepoint, $39 \%$ of the whole sample of patients had received systemic treatment with corticosteroids, and $19.4 \%$ were receiving them at the time of recruitment (prednisone average dose of 6.9 $\mathrm{mg} /$ day and a range of 1.125 to $40 \mathrm{mg}$ ). Accumulated dose of prednisone was higher in patients currently receiving corticosteroids (mean $8605 \mathrm{mg}$ and median $8283 \mathrm{mg}$ ) in comparison to those who had received them in the past (mean $4853 \mathrm{mg}$ and median $2305 \mathrm{mg}$ ).

Fifty one patients were receiving other potentially osteotoxic concomitant treatments. These mainly included methotrexate (received by $5 \%$ of the cohort), sulfasalazine (received by $1 \%$ of the cohort), antiTNF-a alone or in combination with other anti-rheumatic drugs (received by $5 \%$ of the cohort), and other non-anti-TNF-a biologic drugs (received by $2 \%$ of the cohort). Up to $18 \%$ of patients were treated with other immunosuppressants (azathioprine, tacrolimus, mycophenolate), and/or chemotherapeutic agents. There were no patients treated with antiepileptic drugs or blood anticoagulant therapies.

Daily average calcium intake in diet was $696 \mathrm{mg}$. Table 2 shows average daily calcium intake, the daily recommended amount (DRA) [10], and the proportion of patients meeting this criteria by age group. 
Table 2

Daily Mean Calcium Intake

\begin{tabular}{|c|c|c|c|c|c|}
\hline Age Group & $\begin{array}{l}\text { DRA } \\
\text { (mg/day) }\end{array}$ & $\begin{array}{l}\text { Mean Intake } \\
\text { (mg/day) }\end{array}$ & SD & $\begin{array}{l}\text { Range: } \\
\text { min- } \max (\mathrm{mg} / \mathrm{d})\end{array}$ & $\%$ Meeting DRA \\
\hline Pre-school (2-3y) & 700 & 823 & 263 & $513-1346$ & $44.4 \%$ \\
\hline School (4-9y) & 1000 & 655 & 233 & $254-1186$ & $24.2 \%$ \\
\hline Adolescent (10-17y) & 1300 & 695 & 329 & $99-1925$ & $10.9 \%$ \\
\hline Young adult (18-20y) & 1100 & 725 & 156 & $555-985$ & $0 \%$ \\
\hline
\end{tabular}

A decrease in adherence to calcium DRA was observed in accordance with age increase $(p=0.035)$.

The PAQ median and interquartile range by age groups were as follows: School-children median of 3.19 (interquartile range of 0.57 ), adolescents median of 2.8 (interquartile range of 0.95 ), and young adults median of 2.46 (interquartile range of 1.67). A decrease in physical activity associated to older age $(p<0.001)$ was observed.

For $100 \%$ of the cohort, calcemia levels were normal (2.2 to $2.6 \mathrm{mmol} / \mathrm{L})$ with an average of $2.49(0.75)$ $\mathrm{mmol} / \mathrm{L}$. For $86 \%$ of the cohort, phosphatemia levels were normal $(0.96-1.72 \mathrm{mmol} / \mathrm{L})$ with a mean of $1.57(0.21) \mathrm{mmol} / \mathrm{L} .14 \%$ of patients presented elevations of serum phosphorus with a mean of 1.84 $(0.16) \mathrm{nmol} / \mathrm{L}$. Serum calcidiol mean was $66.82(33.65) \mathrm{nmol} / \mathrm{L}$. Calcidiol concentration was normal $(\geq$ $30 \mathrm{nmol} / \mathrm{L}$ ) [11] for $88 \%$ of measurements and poor for $12 \%$, with an average of $22.8(3.9) \mathrm{nmol} / \mathrm{L}$.

Calciuria was determined in $94 \%$ of the cohort. The concentration of calcium in urine was normal in $58.8 \%$ of the cohort, reduced ( $<1.6 \mathrm{mmol} / \mathrm{L}$ ) in $37.1 \%$ of the cohort, and high $(>5.3 \mathrm{mmol} / \mathrm{L})$ in $4.1 \%$ of the cohort. Mean values for the normal, reduced, and high calciuria groups were $2.86(1.1) \mathrm{mmol} / \mathrm{L}, 0.86(0.4)$ $\mathrm{nmol} / \mathrm{L}$ and $7.65(1.8) \mathrm{nmol} / \mathrm{L}$, respectively.

Regarding the RFs, $4.3 \%$ of patients in the cohort sample presented just one risk factor associated to LBM, $38.3 \%$ had 2 RFs, $30.9 \%$ had 3 RFs, $14.9 \%$ had 4 RFs, and $11.7 \%$ had 5 RFs or more. RFs and their frequency in the cohort were:

- Presence of potentially osteopenizing diagnosis (99\%)

- Insufficient dietary calcium intake (84.5\%)

- Drugs with osteotoxic potential (non-steroidal immunosuppressants) (31.1\%)

- Steroid therapy at the time of inclusion in the study (19.4\%)

- Corticosteroids before study inclusion (18.4\%)

- Sedentary lifestyle (PAQ <2) $(13.6 \%)$ 
- History of long bone and vertebral fractures (12.6\%)

- Hypovitaminosis D in blood (<30 nmol/L) (12\%)

- Association to a second potentially osteopenizing diagnosis $(5.8 \%)$

- Hypercalciuria in 24-hour urine (4.1\%)

When comparing the distribution of RFs present in patients with pre-existing conditions, there were no statistically significant differences among diagnoses, except for those patients receiving potentially osteotoxic drugs: immunosuppressants $(p<0.001)$, corticosteroids at the present time $(p<0.001)$ and corticosteroids in the past $(\mathrm{p}=0.050)$, with a higher proportion of corticosteroids in the group of hematologic diseases (75\%), vasculitis (75\%), SADs (74.1\%), and JIA (66.7\%).

Presence of sedentary behaviors, defined as PAQ $<2,(p<0.001)$ and a history of long bone and vertebral fractures $(p=0.004)$ exhibited non-homogeneous distributions. The highest proportion of patients with sedentary behavior was present in the hematologic diseases group (71.4\%), followed by the autoinflammatory diseases group (33.3\%). Regarding patients with fractures, we also found a higher proportion among hematologic diseases (57.1\%), which is followed by the autoinflammatory diseases group (33.3\%) and the digestive diseases group (malabsorption/food allergies) (12.5\%).

As for the number of risk factors associated with each diagnosis group, statistically significant differences were found among groups $(p<0.001)$ : patients suffering from hematologic diseases presented the highest number of RFs (5.4 (1.64)), followed by SADs (3.75 (0.95)), and patients with digestive diseases with the least number of RFs (2.29 (0.7)).

Considering RFs distribution by age groups, statistically significant differences were observed $(p=0.016)$ in distribution of immunosuppressant treatments, with a higher proportion in the adolescent group (43.6\%), followed by young adults (33.3\%), school-aged children (15.2\%), and preschool-aged children (11.1\%). Sedentary lifestyle was a RF also exhibiting a statistically significant difference in its distribution among age groups $(p=0.017)$, present in $33 \%$ of young adults and $20 \%$ of adolescents versus $3 \%$ of school-aged children. As stated above, there were also differences in calcium intake among age groups, but statistically significant differences were not found in the distribution of the rest of RFs.

When evaluating the number of RFs by age group, statistically significant differences in their distribution were also found $(p<0.001)$. Adolescents and young adults presented higher number of RFs (3.4 (1.14) and 3.2 (1.09), respectively), versus school-aged children $(2.3(0.87))$, and preschool-aged children (1.6 $(0.51))$.

Densitometric characteristics of the sample were evaluated. Table 3 shows BMD values for the major body regions of interest by sex. Table 4 shows these values by diagnosis groups. 
Table 3

BMD Values of Major Body Regions of Interest by Sex

\begin{tabular}{|c|c|c|c|c|c|}
\hline & $\begin{array}{l}\text { BMD } \\
\text { TB }\end{array}$ & $\begin{array}{l}\text { BMD } \\
\text { TBLH }\end{array}$ & $\begin{array}{l}\text { Vertebral } \\
\text { BMD }\end{array}$ & $\begin{array}{l}\text { Femur Total } \\
\text { BMD }\end{array}$ & $\begin{array}{l}\text { Femoral Neck } \\
\text { BMD }\end{array}$ \\
\hline \multicolumn{6}{|l|}{ Female } \\
\hline Mean (SD) & $0.83(0.16)$ & $0.71(0.16)$ & $0.69(0.18)$ & $0.74(0.16)$ & $0.69(0.15)$ \\
\hline Range & $0.54-1.17$ & $0.41-1.01$ & $0.39-1.06$ & $0.44-1.17$ & $0.40-10.30$ \\
\hline \multicolumn{6}{|l|}{ Male } \\
\hline Mean (SD) & $0.79(0.16)$ & $0.66(0.18)$ & $0.59(0.16)$ & $0.74(0.17)$ & $0.68(0.14)$ \\
\hline Range & $0.53-1.12$ & $0.39-1$ & $0.36-0.99$ & $0.46-1.06$ & $0.38-0.94$ \\
\hline
\end{tabular}

Table 4

Age-Adjusted BMD of Major Body Regions of Interest by Diagnosis Groups

\begin{tabular}{|llll|}
\hline & $\begin{array}{l}\text { Vertebral BMD } \\
\text { Mean (SD) }\end{array}$ & $\begin{array}{l}\text { BMD TB } \\
\text { Mean (SD) }\end{array}$ & $\begin{array}{l}\text { BMD TBLH } \\
\text { Mean (SD) }\end{array}$ \\
\hline Juvenile idiopathic arthritis (JIA) & $0.70(0.16)$ & $0.86(0.13)$ & $0.73(0.12)$ \\
\hline $\begin{array}{l}\text { Autoinflammatory } \\
\text { diseases }\end{array}$ & $0.76(0.30)$ & $0.94(0.24)$ & $0.78(0.23)$ \\
\hline Vasculitis & $0.74(0.17)$ & $0.91(0.14)$ & $0.80(0.14)$ \\
\hline Connective tissue diseases & $0.83(0.23)$ & $0.93(0.18)$ & $0.82(0.17)$ \\
\hline Malabsorption/ food allergies & $0.66(0.17)$ & $0.84(0.14)$ & $0.72(0.14)$ \\
\hline Hematologic diseases & $0.69(0.16)$ & $0.84(0.16)$ & $0.76(0.15)$ \\
\hline Endocrinopathy & $0.68(0.13)$ & $0.87(0.11)$ & $0.71(0.19)$ \\
\hline BMD TB: BMD total body; BMD TBLH: BMD total body less head; SD: standard deviation \\
\hline
\end{tabular}

When adjusted for age, a higher value of vertebral BMD was observed in females $(p=0.005)$, but it was not observed in total body BMD or in total body less head BMD ( $p=0.762$ and $p=0.902$, respectively).

When evaluating the influence of different diagnoses on age-adjusted BMD of major regions of interest, statistically significant differences were observed of total body BMD $(p=0.009)$, total body less head BMD 
$(p=0.019)$, and vertebral BMD $(p=0.021)$ among groups.

Table 5 shows proportion of LBMca obtained from each diagnosis group for vertebral and total body regions along with their adjusted variants.

Table 5

Proportion of LBMca by Diagnosis Groups Before and After Height Adjustment

\begin{tabular}{|c|c|c|c|c|}
\hline & $\begin{array}{l}\text { LBMca } \\
\text { ver. Z-score } \\
\text { (\%) }\end{array}$ & $\begin{array}{l}\text { LBMca } \\
\text { adj. ver. Z- score } \\
\text { (\%) }\end{array}$ & $\begin{array}{l}\text { LCMca } \\
\text { TB Z-score } \\
(\%)\end{array}$ & $\begin{array}{l}\text { LBMca } \\
\text { adj. TB Z-score } \\
\text { (\%) }\end{array}$ \\
\hline $\begin{array}{l}\text { Juvenile idiopathic arthritis } \\
\text { (JIA) }\end{array}$ & 11.1 & 5.6 & 11.8 & 0 \\
\hline $\begin{array}{l}\text { Autoinflammatory } \\
\text { diseases }\end{array}$ & 0 & 0 & 0 & 0 \\
\hline Vasculitis & 0 & 0 & 25 & 0 \\
\hline Connective tissue diseases & 0 & 0 & 0 & 0 \\
\hline $\begin{array}{l}\text { Malabsorption/ food } \\
\text { allergies }\end{array}$ & 9.1 & 7.1 & 7 & 9.8 \\
\hline Hematologic diseases & 28.6 & 33.3 & 50 & 60 \\
\hline Nephropathies & 0 & 0 & 5.6 & 0 \\
\hline Total & 8.2 & 6.4 & 10.5 & 7.7 \\
\hline $\begin{array}{l}\text { ver. Z-score: vertebral Z-score } \\
\text { body Z-score; adj. TB Z-score } \\
\text { chronological age (Z-score } \leq\end{array}$ & $\begin{array}{l}\text { jj. ver. Z-scol } \\
\text { ight adjuste } \\
\text { D) }\end{array}$ & $\begin{array}{l}\text { leight adjusted v } \\
\text { tal body Z-score }\end{array}$ & $\begin{array}{l}\text { oral Z-score; } \\
\text { Mca: low bo }\end{array}$ & $\begin{array}{l}\text { Z-score: total } \\
\text { nass for }\end{array}$ \\
\hline
\end{tabular}

There were no statistically significant differences of LBMca proportion among diagnosis groups measured by vertebral Z-score $(p=0.263)$, adjusted vertebral Z-score $(p=0.265)$, or total body Z-score $(p=0.157)$. However, there were differences measured by height adjusted total body $Z$-score $(p=0.012)$.

Vertebral morphometry was performed on 95 children: 5 patients showed vertebral fractures, of whom 4 were asymptomatic. 1 female patient had 3 previously known vertebral fractures, and morphometry was performed 1 year after they were diagnosed and discovered 2 additional fractures. The description of the fractures of this patient is as follows: moderate wedge fracture at $\mathrm{T} 7$, mild wedge fracture at $\mathrm{T} 8$, mild wedge fracture at T9, moderate wedge fracture at T12 and severe biconcave L4. Table 6 shows characteristics of children with vertebral fractures found by morphometry. 
Table 6

Fractures by Vertebral Morphometry.

\begin{tabular}{|c|c|c|c|c|c|c|c|}
\hline $\begin{array}{l}\text { Fractures } \\
\mathrm{n}\end{array}$ & $\begin{array}{l}\text { Fracture } \\
\text { Type }\end{array}$ & $\begin{array}{l}\text { Previous } \\
\text { known } \\
\text { Fractures }\end{array}$ & Sex & $\begin{array}{l}\text { Age } \\
\text { (years) }\end{array}$ & Diagnosis & $\begin{array}{l}\text { Other } \\
\text { Fractures }\end{array}$ & LBMca \\
\hline \multirow[t]{3}{*}{5} & $\begin{array}{l}4 \text { wedge } \\
\text { fracture }\end{array}$ & 3 & female & 13 & lymphoma & no & no \\
\hline & $\begin{array}{l}(2 \text { mild and } \\
2 \\
\text { moderated })\end{array}$ & & & & & & \\
\hline & $\begin{array}{l}1 \text { severe } \\
\text { biconcave }\end{array}$ & & & & & & \\
\hline 1 & $\begin{array}{l}\text { T7 mild } \\
\text { wedge } \\
\text { fracture }\end{array}$ & 0 & female & 15 & PAN & no & no \\
\hline 1 & $\begin{array}{l}\text { T7 mild } \\
\text { wedge } \\
\text { fracture }\end{array}$ & 0 & male & 11 & ERA JIA & no & yes \\
\hline 1 & $\begin{array}{l}\text { T8 } \\
\text { moderate } \\
\text { wedge } \\
\text { fracture }\end{array}$ & 1 & female & 11 & $\begin{array}{l}\text { ALL }+ \\
\text { hypopituitarism }\end{array}$ & calcaneus & yes \\
\hline 1 & $\begin{array}{l}\text { T7 mild } \\
\text { biconcave }\end{array}$ & 0 & female & 11 & CMPA & tibia & no \\
\hline
\end{tabular}

A multiple linear regression test was performed in order to evaluate the magnitude of the effect of different risk factors on BMD in major regions of interest. For BMD of total body, there was a statistically significant relationship with age $(p<0.001)$ and sedentary behaviors $(p<0.001)$ with a combined correlation coefficient of $81.9 \%$. Age showed a positive coefficient of +0.034 (BMD per year of age) and sedentary behaviors presented a negative coefficient of -0.085 .

As for total body less head BMD, there was a relationship with age $(p<0.001)$, level of physical activity $(p<0.001)$, and Hispanic ethnicity ( $p=0.031)$. These three factors could explain up to $82.5 \%$ of variability of total body less head BMD, where age added +0.036 per passing year, physical activity subtracted -0.084 , and Hispanic ethnicity subtracted -0.055 .

Regarding vertebral BMD, a statistically significant relationship with age $(p<0.001)$, sex $(p<0.001)$, presence of hypovitaminosis $D(p<0.001)$, and Hispanic ethnicity $(p<0.001)$ was found. They presented a combined correlation coefficient of $73 \%$. Individually, age added +0.032 per each passing year, male sex subtracted -0.061 , hypovitaminosis $D$ added +0.077 , and Hispanic ethnicity subtracted -0.070 . 
There was a statistically significant positive correlation found between the number of risk factors and total body BMD, total body less head BMD, and vertebral BMD with correlation coefficients of $0.43,0.42$, and 0.44 , respectively. However, this relationship was not found when stratified by age.

\section{Discussion:}

The objective of this study was to identify patients at risk of presenting LBMca based on their RFs, to describe this population, and to assess their LBMca and COP prevalence, as well as to study each risk factor's impact on BMD. Patients included in the study were referred to the Pediatric Rheumatology Outpatient Clinic for bone health assessment as they were found to have one or more RFs for developing LBMca. Despite the fact that the majority were referred for only 1 risk factor, we found that more than one third were associated with more than $2 \mathrm{RFs}$, and it is striking that more than one quarter of these patients presented at least 4 RFs.

The most prevalent RF in this study was low calcium intake, which was lower than the recommended calcium intake for $84.5 \%$ of the total cohort. Prior to this study, other studies observed a decrease in calcium intake in young, healthy individuals who are completing the transition to adulthood [12]. A high percentage of inadequate calcium intake favors the presence of additional RFs.

A higher number of RFs was observed in patients with hematologic diseases reaching an average of 4.5 RFs. In addition, the number of RFs increased with age (1.6 in pre-school children, 3.4 in adolescents, and 3.2 in young adults).

When assessing whether there were differences in BMD according to diagnosis group, it was observed that hematologic diseases along with digestive diseases and nephropathies were the groups with the lowest BMD. A BMD decrease in relation to these diagnoses has already been described in the literature, and nephrotic syndrome is one of the most studied. Regarding this syndrome, scientific evidence illustrates that up to $25 \%$ of affected children present Z-scores lower than expected, when compared to an average population, one year after the diagnosis [13].

In this study, the group with the highest proportion of LBMca in all regions was the one with hematologic diseases affecting $60 \%$ of the patients, followed by JIA and digestive diseases.

We observed that RFs related to BMD were age and sedentary lifestyle. In the case of total body BMD there was a contribution up to $81.9 \%$ of the BMD variability, and for total body less head BMD there was a contribution up to $82.5 \%$ along with Hispanic ethnicity. As for vertebral BMD, RFs that explained up to $73 \%$ of variability were age, sex, hypovitaminosis $D$, and ethnicity. The positive effect of hypovitaminosis D could be a potential confounding effect associated with seasonal fluctuations or solar exposition [14, 15], included patient's ethnicity [16], genetic polymorphisms [14, 15, 17], or underlying diseases [15].

It can be deduced from these data that sedentary lifestyle is the most modifiable RF with the highest impact on BMD in pediatric age. In this study, a decrease in level of physical activity linked to age 
increase was observed, and this observation has also been described in a comparable healthy population [6]. The decrease in physical activity level is a growing concern-which is important in both healthy adolescents and adolescents suffering from chronic diseases-especially because of its well-known benefits for both groups $[18,19]$. In this respect, there are studies in which a lower level of physical activity is described in children and adolescents with JIA despite the adequate management of the disease [20]. The same was observed in children suffering from hematologic diseases 10 months after having received their last treatment [21], in children with chronic nephropathies [22], and in children with SADs [23], among others. Our study found a lower level of physical activity in children with hematologic diseases, followed by nephropathies, vasculitis, and autoinflammatory diseases.

A statistically significant relationship was not found when evaluating if the risk factors (stratified by age) influenced total, subtotal less head, and vertebral BMD. However, there was a clear trend of patients with a higher number of risk factors presenting a lower BMD.

This study did not find a statistically significant relationship between many risk factors-or a combination of RFs-and LBMca. This may be linked to the main limitation of this work: the insufficient number of patients in each group, which did not allow the ability to draw statistically significant and conclusive results from the study's subgroups. Consequently, we need to take into account that although these risk factors may not be linked to LBMca in a cross-sectional study, they could be significant over the course of its development, even into adulthood, and predetermine a higher predisposition to osteoporosis in the future. We believe that a long-term longitudinal study of these patients is necessary to assess how bone characteristics evolve over time, as well as to evaluate the persistence of risk factors, many of them modifiable, and their association to LBMca/cOP.

\section{Conclusions:}

In our cohort, LBM prevalence in children with risk factors was up to $10.5 \%$, and prevalence of cOP was $4.85 \%$. The pediatric population who had a risk of LBM/cOP had 2 or more risk factors. Risk factors related with BMD changes were age, sex, sedentary behaviors, ethnicity, and hypovitaminosis $D$. Prospective studies with a higher sample size are needed to assess the effect of different RFs on BMD of a developing bone, as well as their potential to cause future repercussions.

\section{List Of Abbreviations:}

- ALL: acute lymphoblastic leukemia

- BMD: bone mineral density

- BMD TB: bone mineral density of Total Body

- BMD TBLH: bone mass density of Total Body Less Head 
- CMPA: cow's milk protein allergy

- cOP: childhood osteoporosis

- DRA: daily recommended amount

- DXA: dual-energy x-ray absorptiometry

- ERA JIA: enthesitis-related arthritis, a type of juvenile idiopathic arthritis

- JIA: Juvenile Idiopathic Artrhitis

- LBMca: low bone mass for chronological age

- PAN: polyarteritis nodosa

- PAQ-A: Physical Activity Questionnaire for Adolescents

- PAQ-C: Physical Activity Questionnaire for Children

- RF: risk factor

- SADs: systemic autoimmune diseases

- SD: standard desviation

- SLE: systemic lupus erythematosus

- T1D: diabetes mellitus

- ver. Z-score: vertebral Z-score

- adj. ver. Z-score: height adjusted vertebral Z-score

- TB Z-score: total body Z-score

- adj. TB Z-score: height adjusted total body Z-score

\section{Declarations:}

- Ethical Approval and Consent to participate: This study was approved by Fundación de la Gestión sanitaria de l'Hospital de la Santa Creu I Sant Pau ethics committee (NIF G-59.780.494) with the reference code: IIBSP-FRA-2016-11.

- Consent for publication: Informed consent to participate in the study was obtained from all the participants. 
- Availability of supporting data: The datasets during and/or analysed during the current study available from the corresponding author on reasonable request.

- Competing interests: The authors declared no potential conflicts of interest with respect to the research, authorship, and/or publication of this article.

- Funding: This study was not funded.

- Authors' contributions: All authors substantially contributed to the acquisition, analysis or interpretation of data, drafted the manuscript, critically revised the manuscript for important intellectual content, and gave final approval of the version to be published.

- Acknowledgements: We thank the Spanish Rheumatology Society for the original manuscript translation and the SER investigation unit for their methodological support. Thanks also to Hye Sang Park for the review and critical reading of the project.

\section{References:}

1. Osteoporosis prevention, diagnosis, and therapy. NIH Consens Statement, 2000. 17(1): p. 1-45.

2. Pitukcheewanont, P., et al., Bone health in children and adolescents: risk factors for low bone density. Pediatr Endocrinol Rev, 2013. 10(3): p. 318-35.

3. Cooper, C., et al., Review: developmental origins of osteoporotic fracture. Osteoporos Int, 2006. 17(3): p. 337-47.

4. Orozco, P., E. Vilert Garrofa, and M. Zwart Salmeron, Evaluación de la ingesta de calcio en la población adulta de España. Estudio INDICAD. REEMO, 2004. 13(6): p. 117-121.

5. Kowalski, K.C., P.R. Crocker, and R.M. Donen, The physical activity questionnaire for older children (PAQ-C) and adolescents (PAQ-A) manual. 2004.

6. Martínez-Gómez, D., et al., Fiabilidad y validez del cuestionario de actividad física PAQ-A en adolescentes españoles. Revista española de salud pública, 2009. 83: p. 427-439.

7. Zemel, B.S., et al., Height adjustment in assessing dual energy $x$-ray absorptiometry measurements of bone mass and density in children. J Clin Endocrinol Metab, 2010. 95(3): p. 1265-73.

8. Zeytinoglu, M., R.K. Jain, and T.J. Vokes, Vertebral fracture assessment: Enhancing the diagnosis, prevention, and treatment of osteoporosis. Bone, 2017. 104: p. 54-65.

9. Alqahtani, F.F. and A.C. Offiah, Diagnosis of osteoporotic vertebral fractures in children. Pediatr Radiol, 2018.

10. Martinez Suarez, V., J.M. Moreno Villares, and J. Dalmau Serra, [Recommended intake of calcium and vitamin D: positioning of the Nutrition Committee of the AEPJ. An Pediatr (Barc), 2012. 77(1): p. 57.e1-8.

11. Munns, C.F., et al., Global Consensus Recommendations on Prevention and Management of Nutritional Rickets. J Clin Endocrinol Metab, 2016. 101(2): p. 394-415. 
12. Larson, N.I., et al., Calcium and dairy intake: Longitudinal trends during the transition to young adulthood and correlates of calcium intake. J Nutr Educ Behav, 2009. 41(4): p. 254-60.

13. Phan, V., et al., Skeletal findings in the first 12 months following initiation of glucocorticoid therapy for pediatric nephrotic syndrome. Osteoporos Int, 2014. 25(2): p. 627-37.

14. Ferrari, D., G. Lombardi, and G. Banfi, Concerning the vitamin D reference range: pre-analytical and analytical variability of vitamin D measurement. Biochem Med (Zagreb), 2017. 27(3): p. 030501.

15. Doyon, A., et al., Genetic, Environmental, and Disease-Associated Correlates of Vitamin D Status in Children with CKD. Clin J Am Soc Nephrol, 2016. 11(7): p. 1145-53.

16. Rajan, S., T. Weishaar, and B. Keller, Weight and skin colour as predictors of vitamin D status: results of an epidemiological investigation using nationally representative data. Public Health Nutr, 2017. 20(10): p. 1857-1864.

17. Moon, R.J., et al., Response to Antenatal Cholecalciferol Supplementation Is Associated With Common Vitamin D-Related Genetic Variants. J Clin Endocrinol Metab, 2017. 102(8): p. 2941-2949.

18. Marker, A.M., R.G. Steele, and A.E. Noser, Physical activity and health-related quality of life in children and adolescents: A systematic review and meta-analysis. Health Psychol, 2018. 37(10): p. 893-903.

19. Aguilar Jurado, M.A., et al., [Improvement of students' physical condition and health after a physical activity breaks program]. Rev Esp Salud Publica, 2018. 92.

20. Bohr, A.H., et al., Reduced physical activity in children and adolescents with Juvenile Idiopathic Arthritis despite satisfactory control of inflammation. Pediatr Rheumatol Online J, 2015. 13: p. 57.

21. Bianco, A., et al., Evaluation of fitness levels of children with a diagnosis of acute leukemia and lymphoma after completion of chemotherapy and autologous hematopoietic stem cell transplantation. Cancer Med, 2014. 3(2): p. 385-9.

22. Clark, S.L., M.R. Denburg, and S.L. Furth, Physical activity and screen time in adolescents in the chronic kidney disease in children (CKiD) cohort. Pediatr Nephrol, 2016. 31(5): p. 801-8.

23. Houghton, K.M., et al., Fitness, fatigue, disease activity, and quality of life in pediatric lupus. Arthritis Rheum, 2008. 59(4): p. 537-45. 